\title{
Interaction of Convection and Oscillations in the Solar Atmosphere: numerical results
}

\author{
M. Steffen \\ Institut für Theoretische Physik u. Sternwarte \\ Universität Kiel \\ Olshausenstraße 40 \\ D-2300 KIEL 1 \\ West Germany
}

\begin{abstract}
Analysing the results of numerical simulations of solar granular convection we found, superimposed on the convective flow, an oscillatory phenomenon with a well defined period of $260 \pm 15$ sec.
\end{abstract}

\section{INTRODUCTION}

The common approach in studying oscillations of the solar atmosphere is to linearize the basic equations of hydrodynamics which can then be solved if additional simplifying assumptions are made (e.g. undisturbed atmosphere assumed static and isothermal, motions treated as isothermal or adiabatic). In this way the response of such simple systems to external perturbations (thought to be caused by e.g. convective motions) can be investigated (e.g. Meyer u. Schmidt, 1967). In contrast the calculations described below consider in some detail the basic physics governing granular convection. The resulting models are far from being static and isothermal or even polytropic but instead show quite complicated velocity fields and large temperature inhomogeneities. Oscillations are not introduced by external perturbations but are excited naturally durig the time evolution of the system.

\section{CALCULATIONS}

The time-dependent, nonlinear equations of hydrodynamics and radiative transfer describing a compressible flow are solved numerically using the method of bicharacteristics (Stefanik et al., 1984) in order to obtain self-consistent models of granular convection cells. The radiation field is calculated along a large number of rays with various inclinations using realistic Rosseland mean opacities as a function of pressure and temperature (Kurucz, 1979). For the computation of thermodynamic quantities ionization of hydrogen is taken into account. Turbulent viscosity is treated as a free parameter.

A typical model extends vertically over approximately 6 scale heights, from $250 \mathrm{~km}$ below to $650 \mathrm{~km}$ above $\bar{\tau}=1$, with a grid spacing of 20 to $40 \mathrm{~km}$. Horizontal dimensions are varied from 1000 to $2000 \mathrm{~km}$ by changing the horizontal grid width between 15 and $30 \mathrm{~km}$. A total of 
30 (vertical) $x 36$ (horizontal) grid points are used. The time step is typically less than 1 second. The upper boundary is chosen to transmit simple acoustic waves (Stefanik et at., 1984). At the bottom the gas is allowed to flow out of and into the model, requiring the gas pressure to be constant along the lower boundary.

\section{RESULTS}

Assuming axial symmetry, a characteristic stationary flow pattern develops (fig. 1) which extends as high as $300 \mathrm{~km}$ above $\bar{\tau}=1$, distinctly higher than given by local mixing length theory. Maximum velocity occurs in the cool central downdraft. At the top of the hot ascending parts of the flow the temperature gradient is extremely steep (several thousand $\mathrm{K}$ per $100 \mathrm{~km}$ ) due to very efficient cooling by radiation (fig. 2). Considerable horizontal temperature differences are maintained just below the visible surface of such a convection cell. Looked upon from above the model exhibits a bright ring with a dark center, possibly representing an "exploding granule".

Superimposed on this steady convective flow and throughout the upper convectively stable regions of the model we "observe" nearly undamped oscillations with a well defined period of about $260 \pm_{15} \mathrm{sec}$ (fig. 3 ). To a first approximation the oscillatory velocity field can be described as a plane (1-dimensional) vertical motion with amplitude increasing upwards due to a corresponding decrease in density. Closer inspection reveals slight deviations from this picture: small horizontal velocity oscillations also occur and the oscillating flow is not strictly 1-dimensional but clearly reflects the structure of the granule. Maximum velocity amplitude is about $250 \mathrm{~m} / \mathrm{sec}$ at the top of the model $(650 \mathrm{~km})$. The spatial distribution of the temperature oscillations is quite different: the maximum amplitude of $\mathrm{dT}( \pm 190 \mathrm{~K})$ occurs in the regions of steepest temperature gradient. The corresponding periodic variation of the temperature structure is associated with a modulation of the local radiative flux, which is phase-shifted relative to the oscillations of the convective flux by about $90^{\circ}$. The radiative surface flux varies by less than $0.2 \%$.

Test calculations have shown that the period of the oscillations, $P$, is insensitive to the horizontal- and vertical model dimensions, details of the upper and lower boundary condition and details of the numerical scheme. Furthermore it can be ruled out that the opacity and thermal structure of the upper atmosphere $(z \geqq 400 \mathrm{~km})$ determines $P$. The value of the viscosity parameter can be varied without changing the basic oscillation frequency; at very low viscosity additional higher frequencies appear. Amplitude and damping on the other hand are more or less sensitive to all the parameters mentioned above.

\section{CONCLUSIONS}

The period of the oscillations found in our calculations seems to be determined by the internal structure of the mean atmosphere rather than by a cavity related to the external dimensions of the model. A closer look at the spatial properties of the oscillatory component of temperature and radiation field strongly suggests that the driving mechanism is located in the regions where the very large temperature 
gradient occurs. To answer the question what determines the characteristic oscillation frequency and how the driving mechanism really works, more detailed investigations are necessary. The results presented here might be related to the observation that most of the power of the solar oscillations is concentrated in the 5 minute region.
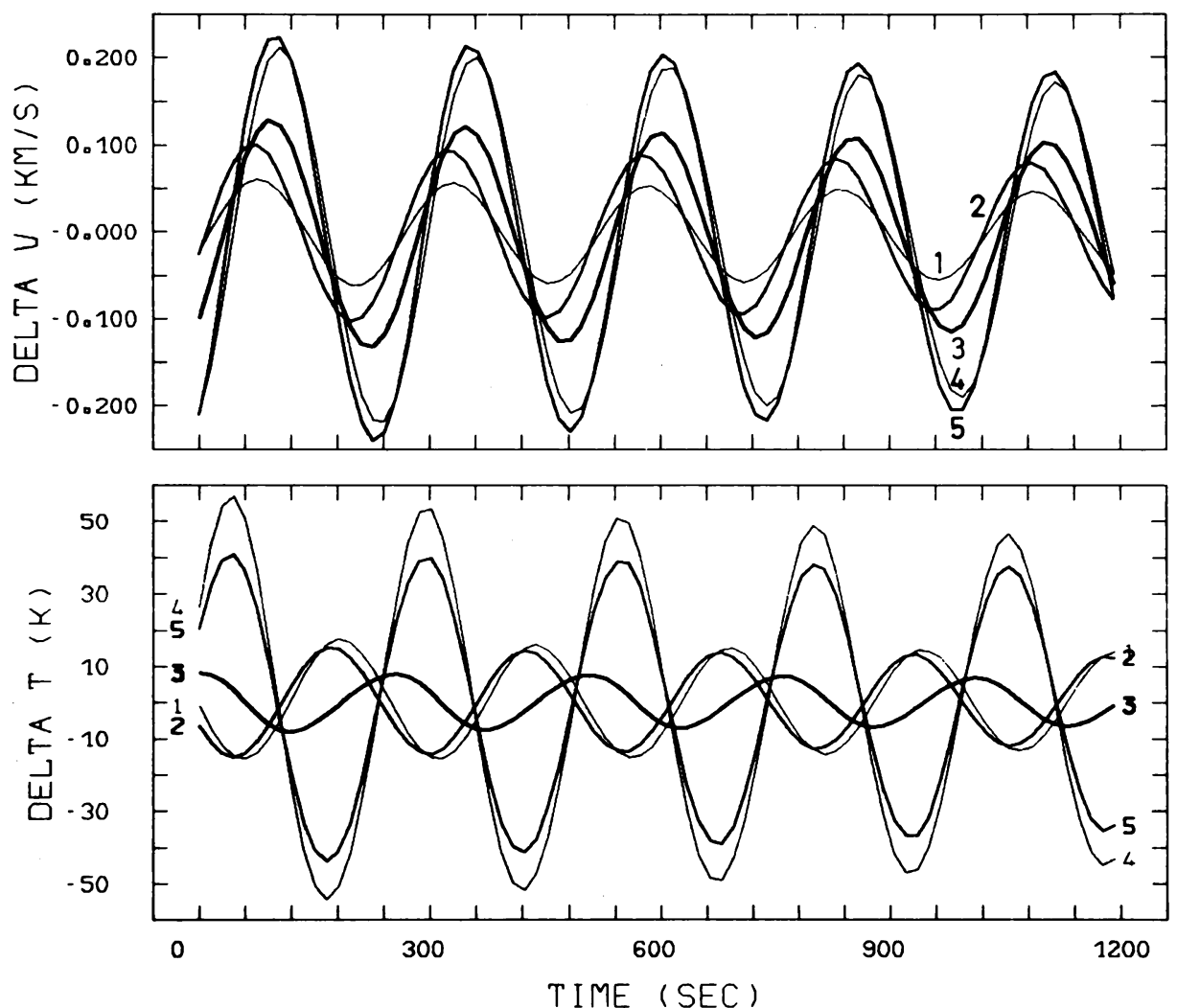

Fig. 3: Superimposed on the steady mean flow shown in fig. 1 and 2 oscillations with a period of about $260 \pm 15 \mathrm{sec}$ are found. The upper panel shows oscillations of the vertical velocity as a function of time at 5 selcted grid points located on the axis of symmetry at heights of $-200,20,200,400$ and $600 \mathrm{~km}$; amplitude is increasing upwards. The lower frame shows the corresponding temperature oscillations. These plots demonstrate that the oscillations are nearly undamped in time and have a well defined period.

\section{REFERENCES}

Kurucz, R.L.: 1979, Astrophys. J. Suppl. 40, 1

Meyer, F., Schmidt, H.U.: 1967, Z. Astrophys. 65, 274

Stefanik, R.P., Ulmschneider, P., Hammer, R., Durrant, C.J.: 1984, Astron. Astrophys. 134, 77 